\title{
Synthesis, characterization, and incorporation of upconverting nanoparticles into a dental adhesive
}

\author{
Rafael Rocha PACHECO(a) (iD) \\ Ali Francisco GARCIA-FLORES(b) (ic \\ Jorge Rodrigo SOTO-MONTERO(c) \\ Guilherme Gorgen LESSEUX(d) (D) \\ Ailla Carla Rocha Acosta LANCELOTT|(e) \\ Eduardo David MARTINEZ(d) (iD \\ Carlos RETTORI(b) (iD) \\ Ricardo Rodrigues URBANO(d) (iD \\ Frederick Allen RUEGGEBERG(f) (iD \\ Marcelo GIANNINI(e) (D) \\ (a) University of Detroit Mercy, Department of \\ Restorative Dentistry, Detroit, MI, USA. \\ (b) Universidade Federal do ABC - \\ UFABC, Center for Natural and Human \\ Sciences, Santo André, SP, Brazil. \\ (c) Universidad de Costa Rica, Faculty of \\ Dentistry, Department of Restorative Sciences, \\ Montes de Oca, San Jose, Costa Rica \\ (d) Universidade Estadual de Campinas \\ - Unicamp, Institute of Physics Gleb \\ Wataghin, Department of Quantum \\ Electronics, Campinas, SP, Brazil. \\ (e) Universidade Estadual de Campinas \\ - Unicamp, Piracicaba Dental \\ School, Department of Restorative Dentistry, \\ Piracicaba, SP, Brazil. \\ (f)Augusta University, Dental College of \\ Georgia, Department of Restorative \\ Sciences, Augusta, GA, USA.
}

Declaration of Interests: The authors of this work report that this research resulted in a Brazilian Patent Aplication N ${ }^{\circ}$ BR 10 2017 027647-3 titled "Nanopartículas com propriedades ópticas de fluorescência e uso"; as well as a U.S. Patent Aplication $N^{\circ} 16 / 228,014$ titled "Fluorescent nanoparticle compositions for dental bonded restorations" filed December 20, 2018.

Corresponding Author:

Marcelo Giannini PhD

E-mail: gianinni@unicamp.br

https://doi.org/10.1590/1807-3107bor-2021.vol35.0120

Submitted: August 11, 2020

Accepted for publication: March 8, 2021

Last revision: Apr 26, 2021

\begin{abstract}
The purpose of this study was to describe the synthesis, characterization, and functionalization of $\mathrm{b}-\mathrm{NaYF}_{4}: 30 \% \mathrm{Yb} / 0.5 \% \mathrm{Tm}$ upconverting nanocrystals for use as nanofillers in a dental adhesive and microscopically evaluate the interface between the particles and a commercial adhesive. The upconverting nanoparticles were synthesized and purified by thermal decomposition, and their chemical composition determined by energy dispersive X-Ray spectroscopy. The crystalline structure was characterized using X-Ray diffraction and morphology and size were observed with scanning and transmission electron microscopy. Upconverting emission was evaluated by spectrophotometry irradiating the particles with a $975 \mathrm{~nm}$ diode laser. Particles were functionalized with polyacrylic acid and the success was confirmed by measurement of Zeta Potential and transmission electron microscopy. The results of X-ray diffraction found a pure hexagonal phase crystalline pattern. Scanning electron microscopy showed uniform dispersion of hexagonal-shaped particles of approximately $150 \mathrm{~nm}$. Upconversion emission was observed in $344 \mathrm{~nm}, 361 \mathrm{~nm}$, $450 \mathrm{~nm}, 474 \mathrm{~nm}, 646 \mathrm{~nm}, 803 \mathrm{~nm}$. Functionalization success was confirmed by formation of a stable aqueous colloid with a Zeta potential of $-29.5 \mathrm{mV}$ and the absence of voids in the particle-adhesive interface on the transmission electron microscopy images. The reported synthesis and functionalization process produced upconverting nanoparticles emitting photons within the blue spectral region (450 $\mathrm{nm}$ and $474 \mathrm{~nm}$ ).
\end{abstract}

Keywords: Nanotechnology; Adhesives; Biomedical and Dental Materials; Spectrum Analysis.

\section{Introduction}

Fluorescent emissions usually follow the Stoke shift principle, where the excitation energy has a higher electron potential (occurs at shorter wavelengths) than the emitted energy (occurring at longer wavelengths). ${ }^{1}$ This effect is usually observed when organic dyes or semiconductor nanocrystals emit visible light under excitation of ultraviolet radiation in a phenomenon known as down-shifting. ${ }^{2,3}$ On the other hand, upconversion (UC) is an anti-Stokes, linear optical process, based on sequential absorption of two or more lower energy, infrared (IR) or near IR photons to an excited state. ${ }^{1}$ A series of light- 
driven transitions excite electrons into higher energy levels, within the $4 \mathrm{f}$ orbital, which then decay radiatively, emitting higher energy, visible photons. ${ }^{1,3}$ Typical upconverting materials consist of an optically inert host ${ }^{4}$ that contains rare-earth (RE) elements such as Nd, Gd, Tb, Ho, Er, Tm and $\mathrm{Yb}$ as dopants. ${ }^{5}$ The UC effect observed for sodium yttrium fluoride $\left(\mathrm{NaYF}_{4}\right)$ crystals doped with $\mathrm{Yb}^{+3}$ and $\mathrm{Tm}^{+3}$ can be described as a three- and four-photon energy transfer upconversion process ${ }^{1}$ resulting in visible light emission between 450 and $500 \mathrm{~nm}$ when exposed to $975 \mathrm{~nm}$ infrared (IR) excitation, ${ }^{3,6,7}$ that has high penetration through materials. ${ }^{2}$

In dentistry, many resin-based restorative materials are activated by visible light at specific wavelengths for excitation of photoinitiators. ${ }^{8}$ Camphorquinone associated with an amine is the most common photoinitiator system for dental applications and is sensitive mainly to blue light having a peak of absorption near $468 \mathrm{~nm} .{ }^{9}$ Light curing is extremely important in restorative dentistry, because polymerization is critical to ensure optimal mechanical properties and clinical longevity of the resultant restoration. ${ }^{10}$ Therefore, the supply of sufficient radiant energy within the absorption range of photoinitiators is essential., ${ }^{911,12}$ However, violet and blue light become highly scattered within different materials resulting in light attenuation within the depths of the materials leading to a limitation known as "depth of cure". ${ }^{12,13,14}$ Limited light penetration trough the materials, results in manufacturers recommending layer placement in 1.5 to $2 \mathrm{~mm}$ thickness for conventional restorative composites, ${ }^{12,15}$ and of 4 to $5 \mathrm{~mm}$ for the newer bulk filled products. ${ }^{16}$ The same light attenuation is observed in indirect restorations where the resin cement is cured through different types and thicknesses of overlying restorative materials. ${ }^{17}$ In either case, light attenuation is associated with a reduction in the degree of conversion and the mechanical properties of polymers activated deep from the restoration surface. ${ }^{12,13}$ Furthermore, during polymerization of dental composites, the free space among monomers is reduced by a volumetric reduction known as polymerization shrinkage, ${ }^{18}$ that is influenced by many factors, including material composition, ${ }^{15}$ cavity preparation design, ${ }^{19}$ filling technique ${ }^{20}$ and, the angle of incidence of the curing light. ${ }^{21}$ Polymerization shrinkage may result in stresses that can affect the integrity of the bonded tooth-restoration interface, ${ }^{21}$ which may lead to internal marginal gap formation, ${ }^{15}$ enamel cracking, ${ }^{22}$ post-operative sensitivity, ${ }^{22}$ marginal staining, and secondary caries. ${ }^{22}$

To overcome the undesired effects, the possibility to initiate the photo-activation by light emitted from an upconverting adhesive layer or liner covering the walls of the preparation, instead of the top surface, could promote peripheral curing and reduce stress development and the formation of internal gaps in the tooth-adhesive-restoration interfaces. ${ }^{21}$ Incorporation of upconverting nanoparticles (UCNPs) in a resin-based cavity liner, adhesive, or cement could result in a material that emits blue and/or violet light when exposed to IR light, ${ }^{3,4}$ and initiate the polymerization reaction from the bonded interface walls. Such emission could change the vectors of polymerization shrinkage stress by producing a low light irradiance or power ${ }^{21}$ irradiating from the peripheral walls of the cavity, ${ }^{21}$ and enhancing the polymerization at depth of the material. ${ }^{23}$ Using a laser source emitting wider wavelength infrared light could reduce the effects of attenuation and scattering ${ }^{24}$ that are reported for violet and blue light. ${ }^{12,25}$ Also, considering the growing applications of low level laser therapy and laser activated photodynamic therapy in dentistry and medicine, ${ }^{26,27}$ the required equipment could be used for multiple purposes.

However, successful incorporation of UCNPs into dental restorative materials must fulfill several requirements. First, the crystallographic phase of the nanocrystal must be controlled, because a hexagonal (b) phase has been reported as the most effective upconverting configuration. ${ }^{1,6}$ Secondly, the size of UCNP should not to be excessively small to avoid surface quenching, nor too large to hinder a homogeneous distribution in the dental resin. Finally, the surface chemistry of the nanofillers must be purified and modified by surface functionalization with appropriate chemical groups to obtain compatibility with the dental resin. A 
previous study reported successful polymerization of a upconverting-particle filled dental adhesive using IR light. ${ }^{7}$ However, the fabrication process relied on large particles that were milled into smaller, irregularly shaped microparticles $(2.8 \mu \mathrm{m}) .^{7}$ Also, the interface between the microparticles and the adhesive material was poor, resulting in cracks and gaps that could produce poor mechanical properties and particle detachment. ${ }^{7}$ Another study showed that incorporation of UCNPs in dental resins resulted in agglomerates, reduced mechanical properties. Also, the reported emission spectrum did not match the absorption peak of camphorquinone, and lacked information on the compatibility between crystals and resin..$^{28}$

For these reasons, the use of smaller and highly luminescent UCNPs with chemically modified surfaces constitutes an innovation aimed at providing homogenous dispersion, improved interface compatibility and a greater ratio of NPs incorporated in the materials while maintaining a constant filler content, allowing IR light sources to activate photopolymerization. The purposes of this study are to describe the synthesis, characterization, and functionalization of $\mathrm{b}-\mathrm{NaYF}_{4}: 30 \% \mathrm{Yb} / 0.5 \% \mathrm{Tm}$ nanocrystals and to analyze the spectral emission from these NPs and incorporate them into a commercial dental adhesive.

\section{Methodology}

\section{Nanoparticle synthesis}

The $\mathrm{NaYF}_{4}: 30 \% \mathrm{Yb} / 0.5 \% \mathrm{Tm}$ nanocrystals were synthesized using the thermal decomposition method. ${ }^{29}$ Ten mL of trifluoroacetic acid (Sigma Aldrich, St. Louis, USA) and $1.16 \mathrm{mg}$ of thulium oxide (Sigma Aldrich, St. Louis, MI,USA) were added in a volumetric, round-bottom flask under magnetic stirring. Under low argon flux, the solution was agitated for 20 minutes at room temperature $\left(23^{\circ} \mathrm{C}\right)$, and then the temperature was gradually increased to $80^{\circ} \mathrm{C}$ and maintained for 30 minutes. The argon flux was increased to evaporate excess trifluoroacetic acid. The resultant solid was thulium trifluoroacetate. After cooling to room temperature, $7.5 \mathrm{~mL}$ of octadecene (Sigma Aldrich, St. Louis,
MI, SA) and $7.5 \mathrm{~mL}$ of oleic acid (Sigma Aldrich, St. Louis, USA) were added into the flask as well as $\mathrm{NaCF}_{3} \mathrm{COOH}$ (Sigma Aldrich, St. Louis, USA), $\mathrm{Y}\left(\mathrm{CF}_{3} \mathrm{COOH}\right)_{3}($ Sigma Aldrich, St. Louis, MI, SA), and $\mathrm{Yb}\left(\mathrm{CF}_{3} \mathrm{COOH}\right)_{3}(\mathrm{GFS}$ Chemicals, Powell, USA). At room temperature, the solution was kept under magnetic stirring and argon flow for 20 minutes to remove oxygen. Then, the temperature was raised to $100^{\circ} \mathrm{C}$ for 30 minutes to remove residual water. After that, the temperature was increased to $330^{\circ} \mathrm{C}$ and maintained for 25 minutes. Finally, the solution cooled to room temperature. ${ }^{30}$

The process resulted in an organic solution where particles were dispersed. ${ }^{31}$ To characterize the particles, and to incorporate them in the dental adhesive, a dry powder was obtained from the solution. Two milliliters of the organic solution were placed into a test tube and $15 \mathrm{~mL}$ of puriss (P.A.) ethanol (Sigma Aldrich, St. Louis, USA) were added, creating an alcoholic solution that was centrifuged for 15 minutes at 3,600 RPM (Excelsa Baby I, Fanem, São Paulo, Brazil), and the supernatant was discarded. The procedure was repeated 3 times. Eight milliliters of chloroform (P.A.) (Sigma Aldrich, St. Louis, USA) were added into the test tube, creating a solution in which the particles were suspended. One milliliter of this solution was added into $2.0 \mathrm{~mL}$ microtubes (Eppendorf Safe-Lock Tube, Eppendorf AG, Hamburg, Germany) and centrifuged for 5 minutes at 12,500 RPM $\left(5^{\circ} \mathrm{C}\right)$ (5810, Eppendorf AG, Hamburg, Germany). The supernatant was discarded, and the excess chloroform was air dried resulting in a dry, white powder of NPs.

\section{Dispersive X-Ray Energy Spectroscopy (EDX)}

To characterize the NP composition, $2 \mathrm{~mL}$ of the chloroform-particle solution were applied on a carbon-tape using a glass rod, mounted on plastic stubs, and sputter-coated with carbon (MED 010, Balzers, Liechtenstein). The samples were evaluated in a scanning electron microscope (SEM) (JSM 5600LV, Jeol, Japan), coupled to an EDX analyzer (Vantage System Noran Instruments, Middleton, USA). ${ }^{2}$ Spectra were obtained with $15 \mathrm{kV}$, 100s lifetime, 20-25\% dead time, at a working distance at $20 \mathrm{~mm}$. 


\section{X-Ray diffraction analyses and Scanning Electron Microscopy (SEM)}

X-Ray diffraction (Phaser D2, Bruker Corp., Billerica, USA) was performed to characterize the crystalline NP structure. The morphology and dispersion of the NPs were evaluated using SEM. The NP suspension prepared for EDX analysis was deposited on a piece of silicon wafer and allowed to dry. NP morphology was observed using a SEM (Quanta 650 FEG, FEI, Hillsboro, USA) operated under $20 \mathrm{kV}$. Images were obtained at 50,000 and 80,000X magnifications. Measurement of the diameter of 200 NPs was performed on the SEM images using a calibrated software (Image 1.52A, National Institute of Health, USA).

\section{Spectral emission}

In a dark room, particles were irradiated using a $975 \mathrm{~nm}$ diode laser (L975P1WJ, Thorlabs Inc., Newton, USA) at $1 \mathrm{~W}$ power, coupled to a diode laser current controller (LDC220C, Thorlabs Inc., Newton, USA) and a thermoelectric temperature controller (TED220C, Thorlabs Inc., Newton, USA). Qualitative emission spectra were obtained using a calibrated spectrophotometer (QEPro, Ocean Optics, Dunedin, USA) associated to a $600 \mu \mathrm{m}$ fiber optic cable. The bare end of the cable was used as detector and an optical filter was used to block the laser excitation light.

\section{Functionalization of UCNPs and incorporation in a dental adhesive}

After synthesis, the external surfaces of the UCNPs were functionalized with polyacrylic acid (PAA) following a previously proposed method for functionalization of hexagonal phase $\mathrm{NaYF}_{4}$ particles. ${ }^{32}$ For the procedure, $171 \mathrm{mg}$ of UCNPs were dispersed in $10 \mathrm{~mL}$ of a $0.1 \mathrm{M} \mathrm{HCl}$ solution and placed in an ultrasonic bath for 1 hour, to remove oleate ligands. The nanoparticles were washed twice with deionized water by centrifugation and redispersion. Extracted particles were dispersed in $15 \mathrm{~mL}$ of a PAA (Polyacrylic acid sodium salt MW $<15,000$, Sigma-Aldrich, St. Louis, USA) aqueous solution $(0.5 \% \mathrm{wt}$.) at $\mathrm{pH} 8$ and kept under vigorous stirring for 1 hour. The colloid was added to $20 \mathrm{ml}$ of diethylene glycol and heated to $100{ }^{\circ} \mathrm{C}$, under stirring, to evaporate water. After two hours, the diethylene glycol colloid was placed in a closed flask and heated in an oven to $130^{\circ} \mathrm{C}$ for 17 hours to ensure the firm bonding between the UCNPs and the PAA ligands. Extraction of the PAA-functionalized UCNPs was performed using centrifugation and redispersion in deionized water three times. Functionalization was considered successful by formation of a stable aqueous colloid ${ }^{32}$ and by Zeta potential measurement (Nanopartica SZ-100 DLS, Horiba Ltd., Kyoto, Japan). Measurement of the Zeta potential of the non-functionalized UCNPs was not performed because they present oleate ligands on the external surface, that make it impossible to form an aqueous dispersion for this analysis. A schematic summary of the functionalization process is presented in Figure 1A.

mass fraction of PAA-UCNP was defined after evaluation of different particle concentrations to determine which adhesive-particle fraction produced the highest upconversion efficiency. A nanofilled (10\% mass fraction of PAA-UCNPs) adhesive was formed by the addition of $10 \mathrm{mg}$ of the functionalized PAA-UCNPs in a 1,5 mL microtube (Eppendorf Safe-Lock Tube, Eppendorf AG, Hamburg, Germany). Then $20 \mu \mathrm{L}$ of chloroform were added to the microtube and the particles were dispersed using vortex agitation for 5 minutes. The density of a commercially available dental adhesive (Adper Single Bond Plus, 3M Oral Care, St. Paul, USA) was measured and resulted in $0.933 \mathrm{~g} / \mathrm{L}$. The adhesive system was selected because it contains a mixture of methacrylate monomers, expected to show an adequate compatibility with the PAA used for functionalization. After dispersion of the UCNPs in chloroform, $110 \mu \mathrm{L}$ of the dental adhesive were added to the microtube and dispersed using vortex agitation and ultrasonic agitation (Cavitron 30K FSI-SLI-10S insert and Cavitron Select SPS ultrasound, Dentsply Professional, PA, SA) for 30 minutes to homogenize the mixture. All the procedures were performed under continuous air flow inside the microtube to evaporate the excess chloroform. A schematic summary of the incorporation of the particles on the adhesive resin is presented in Figure 1B. 


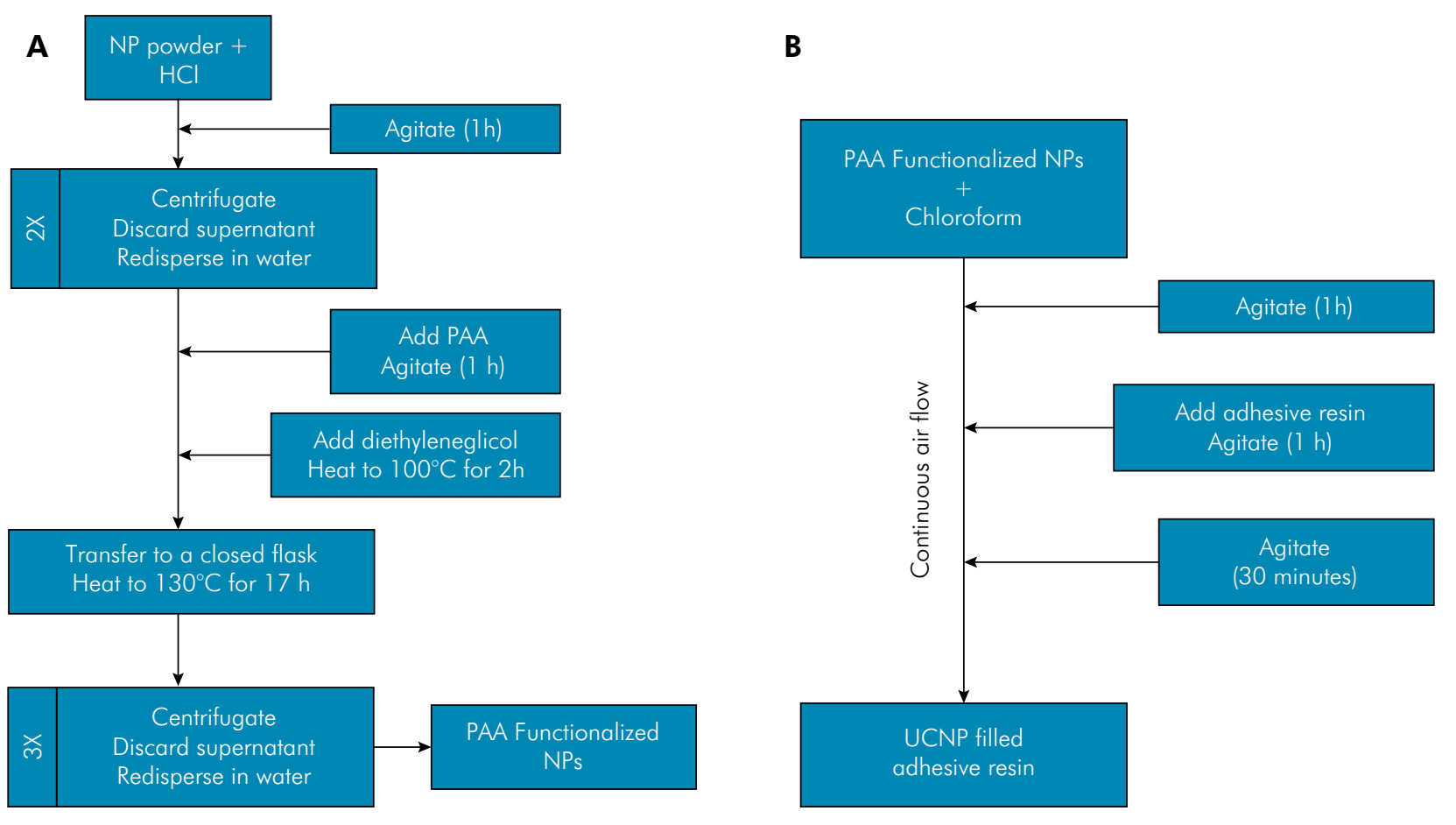

Figure 1. Flowchart summarizing the $(A)$ functionalization and $(B)$ incorporation of the UCNPs into the adhesive resin processes.

\section{Transmission Electron Microscopy (TEM)}

A specimen of each of the un-functionalized and functionalized UCNP-resin mixtures was fabricated. The resins were placed in silicone molds $(15 \times 6 \times 4 \mathrm{~mm})$ and an air jet was applied for 1 minute to evaporate solvents and then light cured using a commercial, multi-peak dental light curing unit (VALO, Ultradent Products Inc., South Jordan, USA emitting $975 \mathrm{~mW} / \mathrm{cm}^{2}$ ) for 20 seconds to obtain nanofilled polymerized blocks. Thin sections $(80 \mathrm{~nm})$ of these blocks were obtained using an ultramicrotome (EM UC6, Leica Microsysteme $\mathrm{GmbH}$, Viena, Austria) and collected on 200-mesh carbon/formvar-coated copper grids (EMS, Hatfield, USA). The prepared specimens were evaluated in a transmission electron microscope (TEM) (JEM 1400, Jeol, Tokyo, Japan, operated at $80 \mathrm{kV}$ ) at 100,000 and 300,000X magnification.

\section{Results}

resulted in an organic solution that underwent a purification method resulting in isolation of a fine, white powder. Figure 2 represents the EDX spectrum of the UCNPs, identifying elements present in the final product (b-NaYF $4: 30 \% \mathrm{Yb} / 0.5 \% \mathrm{Tm})$ : $\operatorname{sodium}(\mathrm{Na})$, yttrium $(\mathrm{Y})$, ytterbium $(\mathrm{Yb})$, chlorine $(\mathrm{Cl}),(\mathrm{Ca})$, and fluorine $(\mathrm{F})$. Because the $\mathrm{X}$-ray emission lines from $\mathrm{Tm}$ and $\mathrm{Yb}$ are too close to separate (La: 7.179 and $7.414 \mathrm{keV}$ and M: 1.462 and $1.521 \mathrm{keV}$, respectively) it is likely that the emission lines appear superimposed, and the much higher concentration of Yb blocks the signature from $\mathrm{Tm}$.

X-Ray diffraction characterized the crystalline structure of the UCNPs as a pure hexagonal-phase pattern (b-NaYF ${ }_{4}, \mathrm{P}-6_{3} / \mathrm{m}$ space group), tabulated as COD1517672 (Crystallography Open Database) code. The diffractogram with the indexed peaks is shown in Figure 3. Morphological analysis of the synthesized particles using SEM showed a uniform dispersion of hexagonal, plate-shaped nanocrystals (Figure $4 \mathrm{~A}$ and B). From the particle size distribution (Figure 4C), it can be noted that the particles vary in size from 100 to $210 \mathrm{~nm}$, with most particles falling in the range between 130 and $170 \mathrm{~nm}$, and a mean diameter of approximately $150 \mathrm{~nm}$.

The emission spectrum of $\mathrm{b}-\mathrm{NaYF}_{4}: 30 \% \mathrm{Yb} /$ $0.5 \%$ Tm under $975 \mathrm{~nm}$ IR excitation is displayed in 
Figure 5. Upconversion emission in the blue light region $(450 \mathrm{~nm}$ and $474 \mathrm{~nm}$ ) and other light emissions at $344 \mathrm{~nm}$ and $361 \mathrm{~nm}$ (ultraviolet) can be seen. Emissions at $646 \mathrm{~nm}$ (red) and at $803 \mathrm{~nm}$ (near infrared) were also observed. Measurement of the Zeta potential resulted in a value of $-29.5 \mathrm{mV}$ (Figure 6). TEM images show hexagonal-shape UCNPs, incorporated to the commercial bonding agent. In the non-functionalized

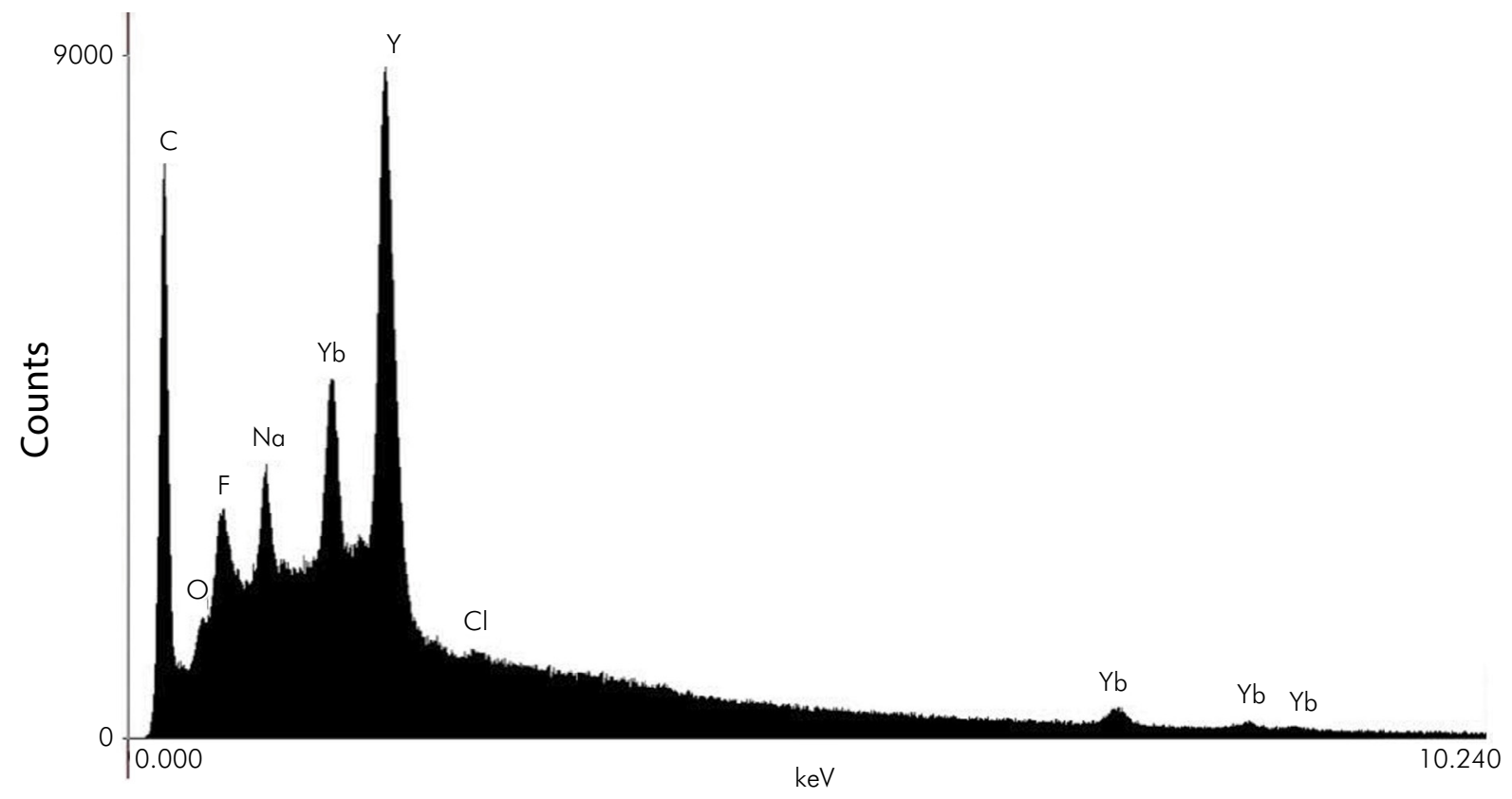

Figure 2. EDX spectrum of the synthetized UCNPs and compositional analysis. The peaks indicate the presence of Carbon Oxygen, Fluorine, Sodium, Ytterbium, Yttrium, and Chlorine elements resulting from the particles synthesis.

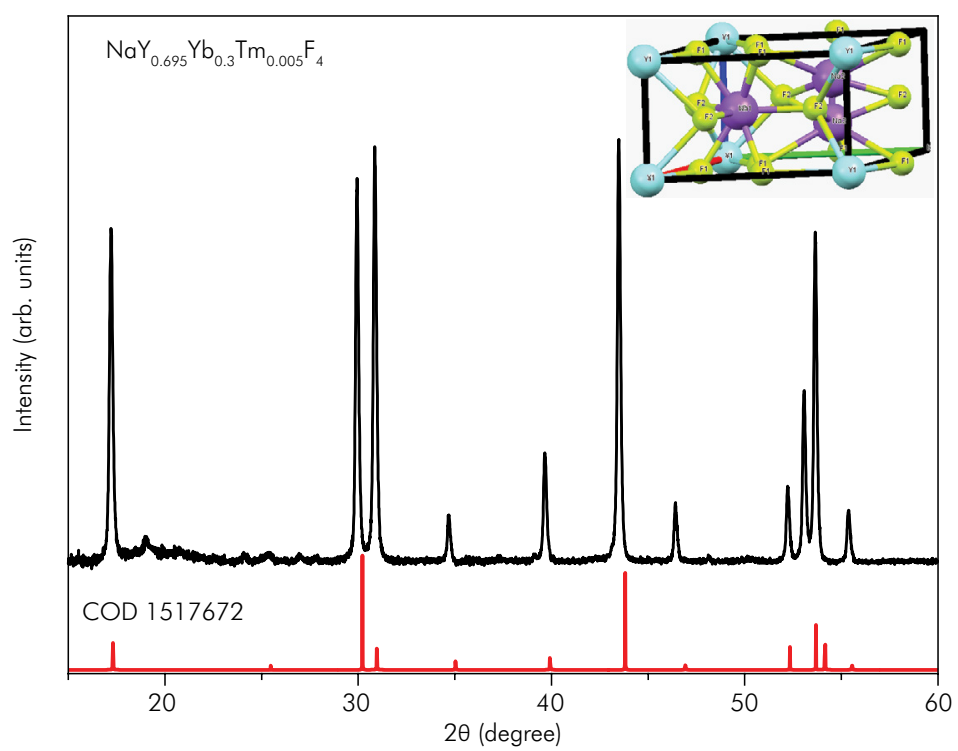

Figure 3. X-Ray diffraction pattern performed on $b-\mathrm{NaYF}_{4}: 30 \% \mathrm{Yb} / 0.5 \% \mathrm{Tm}$ synthetized NPs. Inset: crystal structure representation of $\mathrm{b}-\mathrm{NaYF}_{4}$ (COD 1517672). The spectral profile is presented as the bottom, red line. Lanthanide dopants are in the atomic sites $\mathrm{Nal}$ and $\mathrm{Y} 1$. 

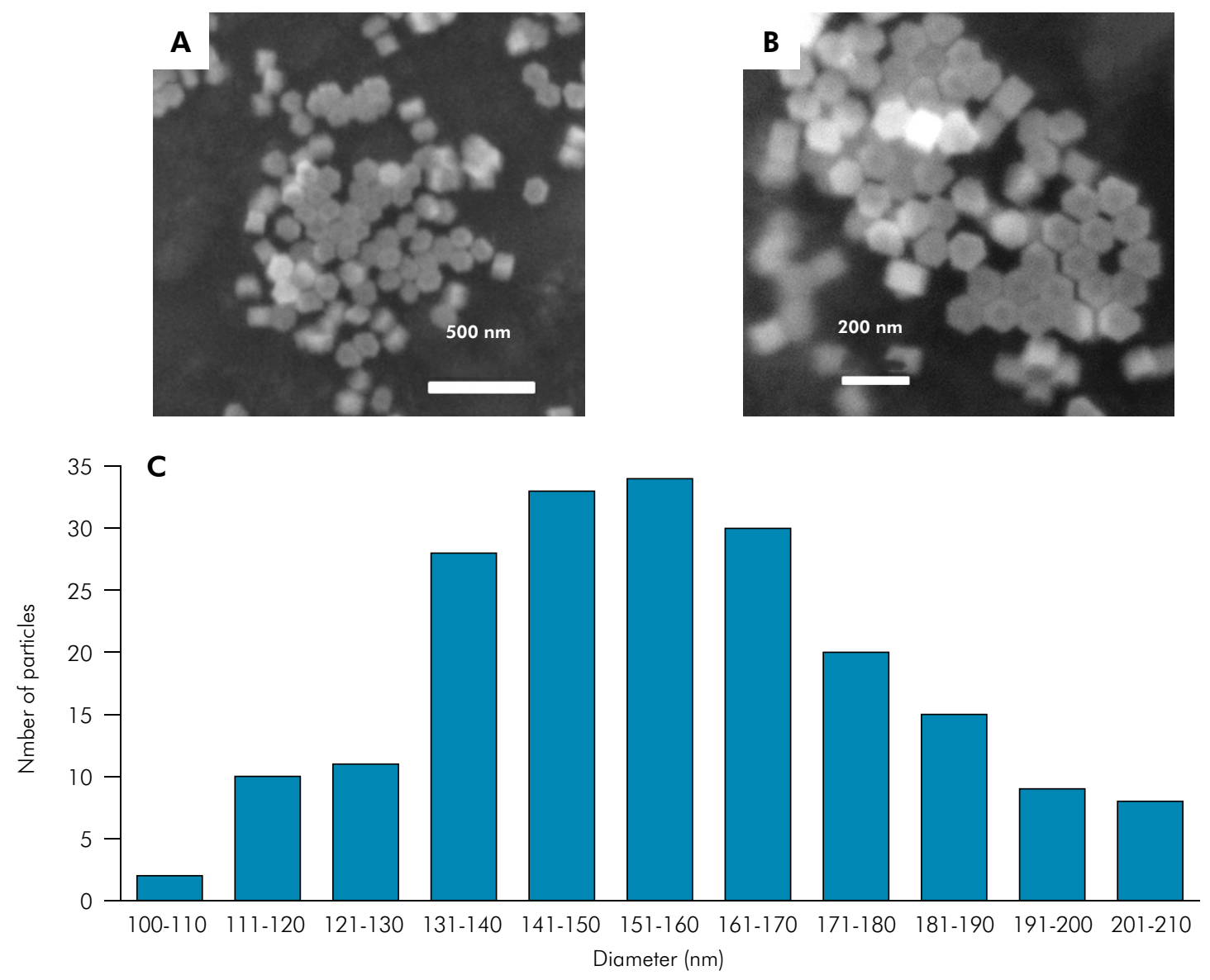

Figure 4. $\mathrm{SEM}$ images of $\mathrm{b}-\mathrm{NaYF}_{4}: 30 \% \mathrm{Yb} / 0.5 \% \mathrm{Tm}$ synthetized NPs at (A) $100,000 \mathrm{X}$ and (B) $150,000 \mathrm{X}$ magnification. (C) Histogram of the particle sizes obtained from SEM images of $200 \mathrm{NPs}$.

sample, NPs are isolated from the adhesive resin matrix, showing voids between the adhesive resin and the particles, also it can be noticed the formation of agglomerates (Figure 7). After functionalization, the surfaces of the UCNPs seems to be well-wetted by the resin matrix, and there are no indications of resin voids (Figure 8).

\section{Discussion}

Analysis using EDX confirmed the expected composition for the synthesized UCNPs, while X-Ray diffraction validated the presence of the desired hexagonal, $\beta-\mathrm{NaYF}_{4}$ crystalline phase. SEM and TEM images characterized the particle shape and size. The synthesis resulted in a viscous, brown organic solution that required purification. The results are in line with other studies that reported uniform dispersion of hexagonal-shaped nanocrystals using similar synthesis methods. ${ }^{6,30}$ It has been reported that the $\mathrm{NaYF}_{4}$ is efficient to produce upconverting emission after doping with $\mathrm{Yb},{ }^{3,5}$ and handling the concentration and type of dopant RE elements allows to obtain a required emission spectrum. ${ }^{3,33}$ In the case of the synthetized UCNPs, the dopant $\mathrm{RE}$ as well as their concentrations were formulated to obtain a three or four -photon energy transfers, that resulted in the upconverting emission of blue, violet and ultraviolet light. It is worth mentioning that the chemical element Tm was not detected in the EDX analysis due to its low concentration (0.5\%) and the difficulty to differentiate it from the other rare earth $\mathrm{Yb}$.

Control of the synthesis process to obtain single phase UCNPs is critical, because the hexagonal crystals display higher UC efficiency, compared 
to cubic crystals. ${ }^{29,30}$ Synthesis of the UC particles resulted in uniform, $150 \mathrm{~nm}$ particles with similar size to that of the inorganic portion of some commercial, nanofilled resin composites. Further analysis should evaluate the viability of developing an experimental resin-based composite consisting of di-methacrylate

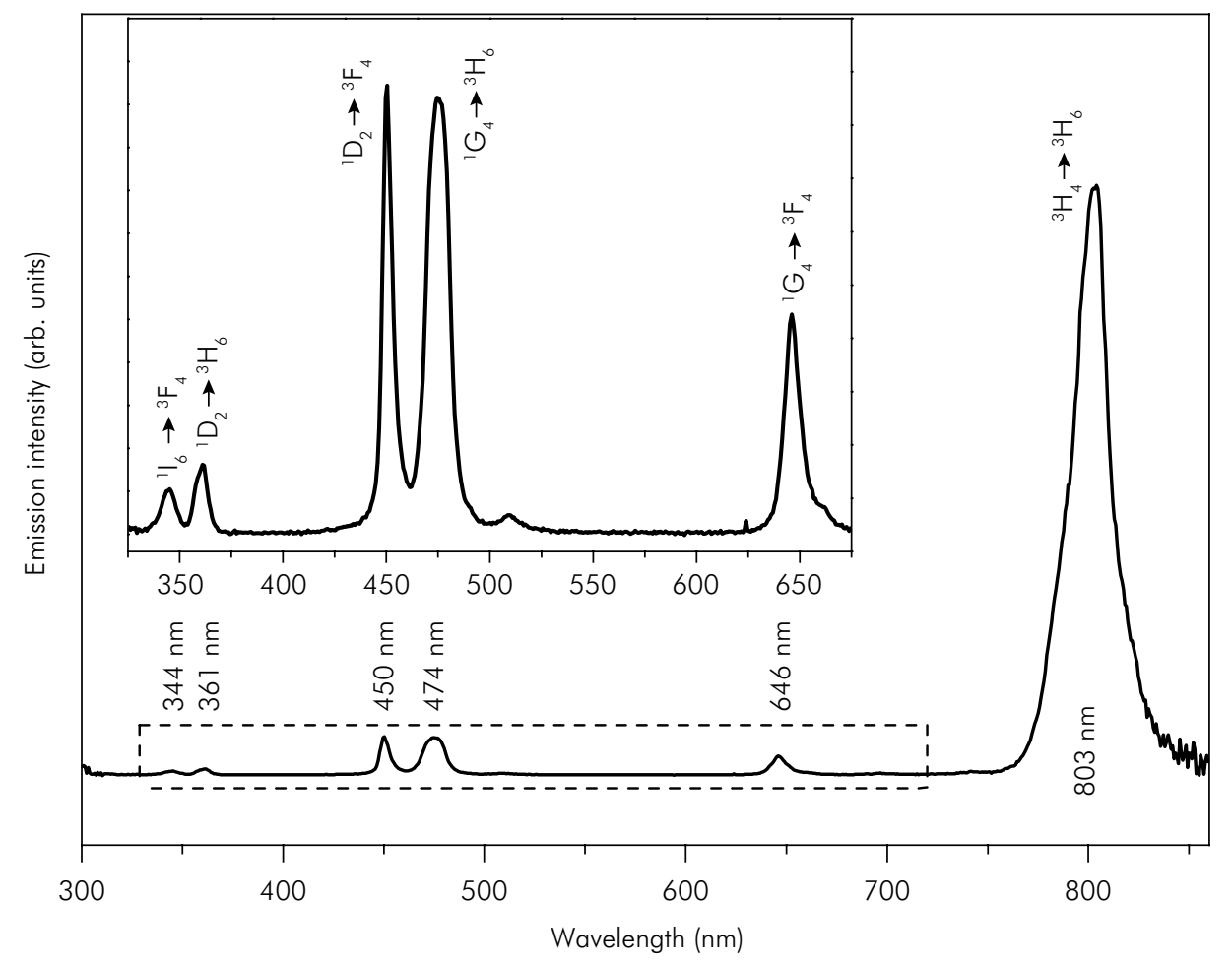

Figure 5. Emission spectrum of the $\mathrm{b}-\mathrm{NaYF}_{4}: 30 \% \mathrm{Yb} / 0.5 \% \mathrm{Tm}$ UCNP powder under $975 \mathrm{~nm}$ diode laser excitation at $200 \mathrm{~mW}$ and 15 seconds acquisition time. Notice peaks at $450 \mathrm{~nm}\left({ }^{1} D_{2}-{ }^{3} F_{4},{ }^{1} D_{2}\right.$ indicates an energy level of the $\mathrm{Tm}^{3+}$ ion and ${ }^{1} D_{2}-{ }^{3} F_{4}$ indicates an energy transition.) and $474 \mathrm{~nm}\left({ }^{1} \mathrm{G}_{4}-{ }^{3} \mathrm{H}_{6}\right)$, a wavelength of optimum absorption for the dental photoinitiator, camphorquinone.

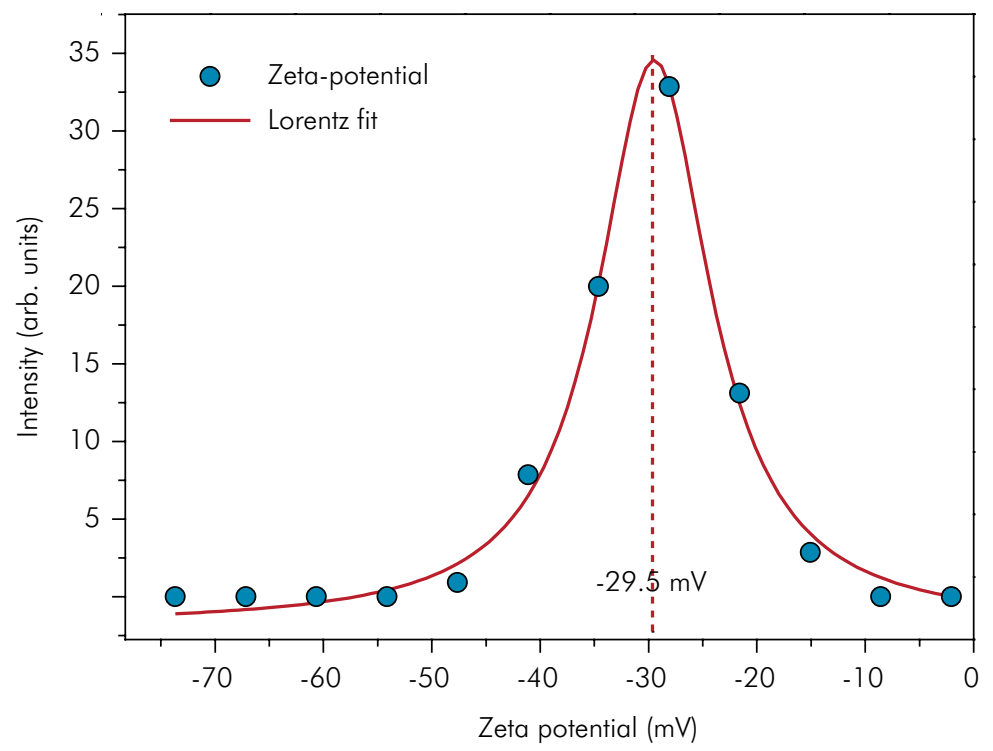

Figure 6. Zeta potential measurement of UC NP functionalized with PAA. 

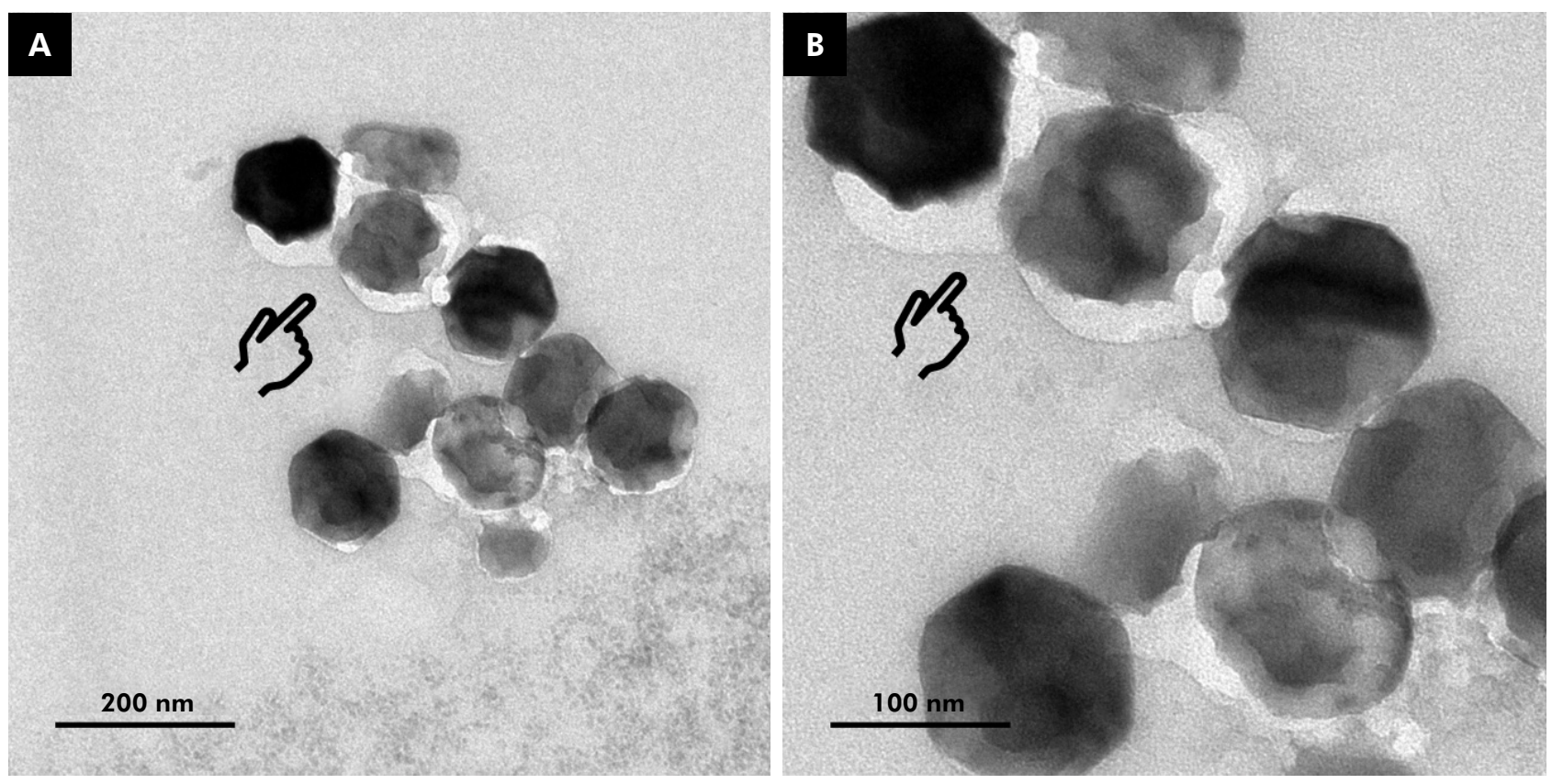

Figure 7. TEM image at of the non-functionalized UCNPs incorporated into the dental adhesive at (A) 100,000X, and (B) 300,000X magnification. Notice the generalized presence of voids indicated by the pointer, and the inability of the cured resin matrix to coat the particles.
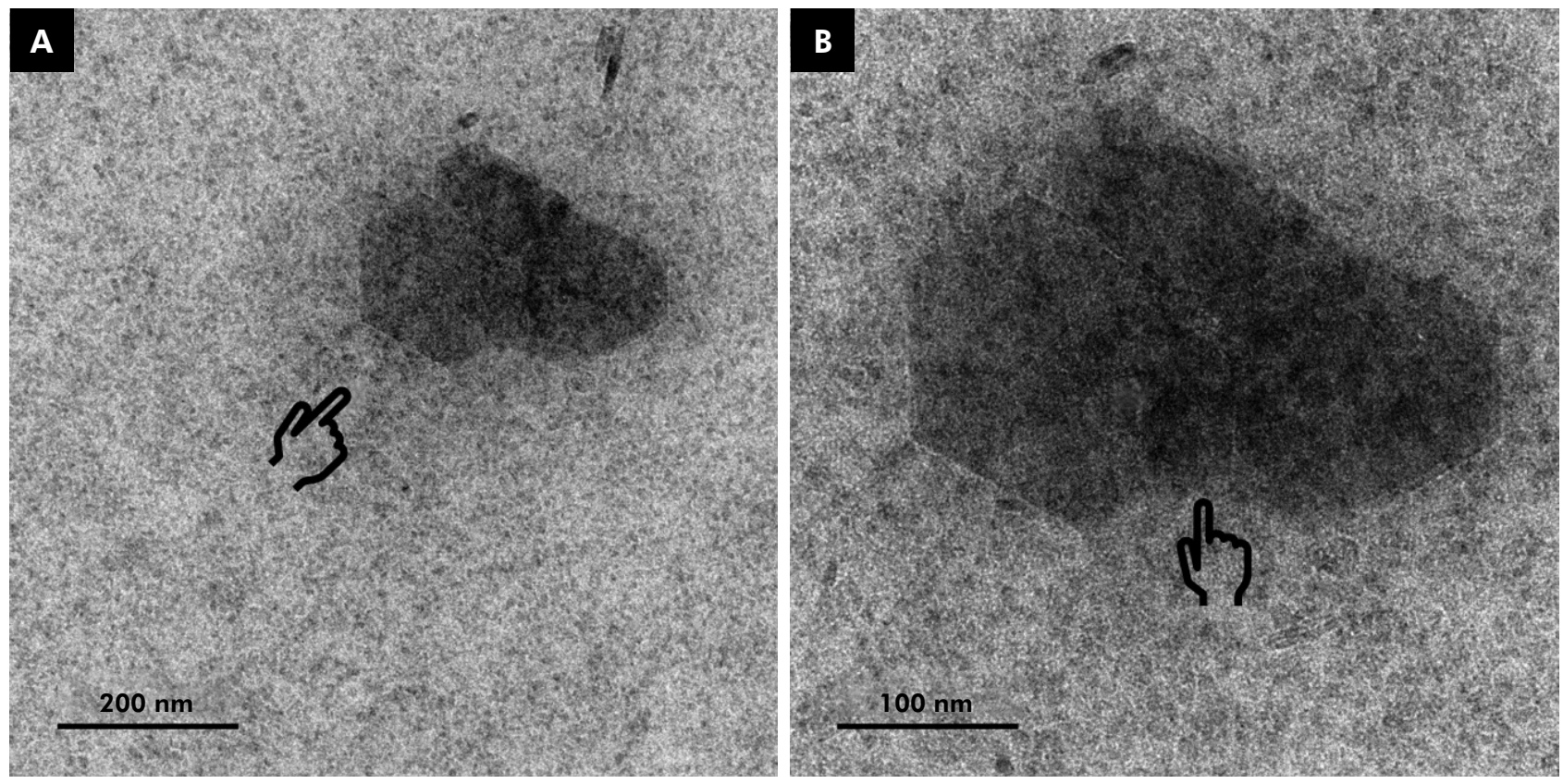

Figure 8. TEM image at of the functionalized UCNPs incorporated into the dental adhesive at (A) 100,000X, and (B) 300,000X magnification. Note the absence of voids indicated by the pointer and the intimate approximation (wetting) of the resin matrix on the surface of the functionalized particles. 
monomers and upconverting inorganic fillers. This experimental composite must be tested regarding the degree of conversion and marginal adaptation of restorations after complementary activation using an IR laser source and a conventional LCU to prove if this technology provides benefits to the restorative procedure. A previous study compared the mechanical properties of a composite resin containing $\mathrm{Y}_{2} \mathrm{O}_{3} \mathrm{NPs}$ co-doped with $\mathrm{Tm}^{3+}$ and $\mathrm{Yb}^{3+}$ as filler, with those obtained using a commercial filler, while also studying a potential light curing by IR excitation. ${ }^{28}$ The shape of the UCNPs appeared hexagonal, and IR excitation at $980 \mathrm{~nm}$ produced emission of blue photons near $480 \mathrm{~nm}$. However, the NPs were agglomerated into porous microstructures of 10 to $40 \mu \mathrm{m}$ diameter clusters. They reported adequate results for Young's modulus, but not for hardness when the experimental UCNP-filled composite was compared with a commercial one.

Another study synthetized hexagonal-shaped, sodium yttrium fluoride co-doped with ytterbium and thulium (NaYF4:25\%Yb3+,0.3\% Tm3+), which was used as a UC phosphor. ${ }^{7}$ Microcrystalline particles of this pure hexagonal phase were prepared using a solid-state synthesis method resulting in quite large particles: 20 to $30 \mu \mathrm{m}$. The results indicated that composite containing the UC particles and exposed to a $980 \mathrm{~nm}$ IR laser showed shorter curing times and higher degree of conversion in comparison to curing with a by a blue LED light. In addition, that study also showed deeper light transmission of IR through tooth tissues than that of blue light (450 $\mathrm{nm}) .{ }^{7}$ However, it has been reported that formation of large particles or clusters above $1 \mathrm{~mm}$ reduces the total active area of the upconverting fillers and might reduce the effective radiant emittance obtained from upconverting particles. ${ }^{34}$

The increased surface area obtained from the reported technique for synthesis of unground, non-clustered, nano-sized (150 nm), UCNPs could render a larger emitting surface and higher quantum efficiency than would micro-sized or clustered particles. ${ }^{35}$ Also, the presented functionalization process has been reported to reduce particle aggregation and agglomeration, ${ }^{32}$ and along with the exchange of the oleate ligands on the outer surface of the UCNPs for PAA ligands, ${ }^{32}$ would work as a wetting enhancing mechanism of the UCNPs by the methacrylate monomers used in several dental materials. The ability to enhance UCNP wetting and thus the intimacy of contact between the particle and its embedding resin matrix would also help to strengthen that material, as well as the bonded joint at the resin/tooth interface. Previous studies report UCNPs dispersed in a chloroform solution, ${ }^{30,36}$ however, because the particles should be as pure as possible for incorporation into resin-based materials, a controlled synthesis and purification process is required to obtain viable UCNPs. The incorporation of $>1 \mathrm{~mm} \mathrm{NaYF}_{4} \mathrm{UCNPs}_{\text {into a resin- }}$ based material has been reported in the literature with major differences to what is proposed in the present study, ${ }^{7}$ because a restorative UCNP-filled material would emit blue-light from within the whole composite increment, ${ }^{7}$ still leading to the problems of polymerization shrinkage produced by conventional blue-light curing. ${ }^{21}$

However, the proposed incorporation of UCNPs into a material that covers the peripheral cavity walls, such as a nano-filled liner/base, adhesive, or resin cement could result in blue light emitted from the preparation walls, leading to an initial polymerization of the adjacent material, that would afterwards be completed by conventional light curing. This protocol might reduce the effects of polymerization shrinkage stress and improve the internal adaptation of the composite to the cavity walls, by producing an initial polymerization of the adjacent material, that would resist the stresses generated during the conventional light curing cycle, ${ }^{18,21}$ while also having the potential to achieve a greater depth of cure on the overlying material, because the curing light would be irradiated from the peripheral cavity walls, instead of only from the cavo-superficial opening of the cavity. In that regard, the successful incorporation of the UCNPs into a dental adhesive can be considered a first step towards the formulation new kind of liner with upconverting properties that could enhance the polymerization at depth of light curable restorative materials, or cements that can be light cured through thick indirect restorations. In those scenarios, the transmission of the IR light 
through dental restorative materials as well as the IR light transmission through different dental substrates should be evaluated in future studies. Also, the influence of different monomeric matrixes must be examined because a different medium might inhibit the upconverting effect. In the present study, the UCNPs were linked to the organic matrix of an adhesive by surface functionalization with PAA, which was confirmed by colloid stability and the Zeta potential value of $-29.5 \mathrm{mV} .32$ However, the durability and enhanced compatibility of the proposed functionalization method in the UCNPs also needs further evaluation with more complex methodologies such as thermogravimetric analysis.

Upconversion from the synthetized b-NaYF $30 \% \mathrm{Yb} / 0.5 \% \mathrm{Tm}$ UCNPs when irradiated with a 975 $\mathrm{nm}$ IR laser produced emission peaks at $450 \mathrm{~nm}$ and $474 \mathrm{~nm},{ }^{4}$ that are optimal for camphorquinone excitation and interestingly similar to the spectral emission observed in dental light curing units. ${ }^{11,12,37}$ Also, light emissions at $344 \mathrm{~nm}$ and $361 \mathrm{~nm}$ are adequate to excite the more quantum efficient, Norrish Type 1 photoinitiators, sensitive to excitation in the ultra-violet spectral region below $390 \mathrm{~nm}$, such as Lucirin TPO (2,4,6-trimethylbenzoyldiphenylphosphine oxide), PPD (1-phenyl-1,2-propanedione), and Ivocerin, ${ }^{11}$ frequently used in modern day composites, that are currently activated by using multiple-peak light curing units. ${ }^{10,12}$ These alternative photoinitiators, are more efficient in generating free-radicals and thus require lower energy doses to form such radicals compared to that of camphorquinone. ${ }^{10,15}$ Even though Type I photoinitiators are known for being more efficient, violet light has a shorter wavelength than blue light, and suffers a higher scattering effect, reducing the depth of penetration. ${ }^{13}$ Regarding the use of a laser source for excitation of the UCNPs, the acquisition of a IR laser could offer new therapeutic alternatives for the clinician, using it also for photobiomodulation, photodynamic therapy, or esthetic procedures. ${ }^{26,27,38}$

Lastly, it must be considered, that despite the promissory advances that this paper presents for the improvement of internal adaptation to cavity walls and the polymerization at depth of light-curable dental materials, further studies are required to validate the potential benefits of this technology. This technique report is a proof of concept to share the successful synthesis and functionalization of the UCNPs, as well as the incorporation into a methacrylate-based dental adhesive. Future research should report the synthesis of the proposed resinous cavity liner and evaluate the depth of cure, marginal adaptation and mechanical properties of restorations using that cavity liner for the early polymerization of the adjacent restorative material. Also, tuning the dopant RE elements could increase the emission efficiency of violet light to activate photoinitiators sensitive to this spectral range, and thus increase the localized peripheral light emission produced by the UCNPs, compared to irradiation from the top of the cavity, using violet LEDs. In this situation, the IR light used to activate the UCNPs would have greater penetration compared to the conventional blue and blue/violet LED-based light curing units. In addition, using praseodymium ions $\left(\mathrm{Pr}^{+3}\right)$ could lead to a three-photon effect, which would upconvert from $477 \mathrm{~nm}$ (blue light) excitation to ultraviolet mission. ${ }^{1,39}$

\section{Conclusions}

Within the limitations of this report, it can be concluded that the synthesized nanoparticles demonstrated upconverting emission at four wavelengths of clinical relevance in dentistry $(344 \mathrm{~nm}$, $361 \mathrm{~nm}, 450 \mathrm{~nm}$, and $475 \mathrm{~nm}$ ) when excited using a $975 \mathrm{~nm}$ IR laser. Also, the functionalization of upconverting particles with polyacrylic acid allowed to obtain a void-free, intimate contact of the synthetized UCNPs with the polymer matrix of a commercially available dental adhesive. Further evaluation is required to determine the industrial and biological viability of incorporating UCNP in resin-based restorative materials.

\section{Acnowledgment}

This study was supported by the Coordenação de Aperfeiçoamento de Pessoal de Nível Superior Brasil (Capes - Finance Code 001 and grants \#A0432013 and \#1777/2014), by grants \# 2011/19924-2, 2012/04870-7 and 2012/05903 of the São Paulo Research Foundation (Fapesp) and by grant \#OAICE047-2017 of the University of Costa Rica. 
Synthesis, characterization, and incorporation of upconverting nanoparticles into a dental adhesive

\section{References}

1. Auzel F. Upconversion and anti-Stokes processes with $\mathrm{f}$ and d ions in solids. Chem Rev. 2004 Jan;104(1):139-73. https://doi.org/10.1021/cr020357g

2. Li Z, Zhang Y. An efficient and user-friendly method for the synthesis of hexagonal-phase $\mathrm{NaYF}(4): \mathrm{Yb}, \mathrm{Er} / \mathrm{Tm}$ nanocrystals with controllable shape and upconversion fluorescence. Nanotechnology. 2008 Aug;19(34):345606. https://doi.org/10.1088/0957-4484/19/34/345606

3. Huang H, Chen J, Liu Y, Lin J, Wang S, Huang F, et al. Lanthanide-Doped Core@Multishell Nanoarchitectures: Multimodal Excitable Upconverting/Downshifting Luminescence and High-Level Anti-Counterfeiting. Small. 2020 May;16(19):e2000708. https://doi.org/10.1002/smll.202000708

4. Huang $H$, Huang F, Lin L, Feng Z, Cheng Y, Wang Y, et al. Perceiving linear-velocity by multiphoton upconversion. ACS Appl Mater Interfaces. 2019 Dec;11(49):46379-85. https://doi.org/10.1021/acsami.9b17507

5. Zhou B, Shi B, Jin D, Liu X. Controlling upconversion nanocrystals for emerging applications. Nat Nanotechnol. 2015 Nov;10(11):924-36. https://doi.org/10.1038/nnano.2015.251

6. Boyer JC, Cuccia LA, Capobianco JA. Synthesis of colloidal upconverting NaYF4: Er3+/Yb3+ and Tm3+/Yb3+ monodisperse nanocrystals. Nano Lett. 2007 Mar;7(3):847-52. https://doi.org/10.1021/nl070235+

7. Stepuk A, Mohn D, Grass RN, Zehnder M, Krämer KW, Pellé F, et al. Use of NIR light and upconversion phosphors in light-curable polymers. Dent Mater. 2012 Mar;28(3):304-11. https://doi.org/10.1016/i.dental.2011.11.018

8. Ferracane JL. Resin composite: state of the art. Dent Mater. 2011 Jan;27(1):29-38. https://doi.org/10.1016/i.dental.2010.10.020

9. Stansbury JW. Curing dental resins and composites by photopolymerization. J Esthet Dent. 2000;12(6):300-8. https://doi.org/10.1111/j.1708-8240.2000.tb00239.x

10. Price RB, Shortall AC, Palin WM. Contemporary issues in light curing. Oper Dent. 2014 Jan-Feb;39(1):4-14. https://doi.org/10.2341/13-067-LIT

11. Soto-Montero J, Nima G, Dias CT, Price RB, Giannini M. Influence of beam homogenization on bond strength of adhesives to dentin. Dent Mater. 2021 Feb;37(2):e47-58. https://doi.org/10.1016/i.dental.2020.10.003

12. Soto-Montero J, Nima G, Rueggeberg FA, Dias C, Giannini M. Influence of multiple peak light-emitting-diode curing unit beam homogenization tips on microhardness of resin composites. Oper Dent. 2020 May/Jun;45(3):327-38. https://doi.org/10.2341/19-027-L

13. Price RB, Rueggeberg FA, Harlow J, Sullivan B. Effect of mold type, diameter, and uncured composite removal method on depth of cure. Clin Oral Investig. 2016 Sep;20(7):1699-707. https://doi.org/10.1007/s00784-015-1672-4

14. Romano BC, Soto-Montero J, Rueggeberg FA, Giannini M. Effects of extending duration of exposure to curing light and different measurement methods on depth-of-cure analyses of conventional and bulk-fill composites. Eur J Oral Sci. 2020 Aug;128(4):336-44. https://doi.org/10.1111/eos.12703

15. Fronza BM, Rueggeberg FA, Braga RR, Mogilevych B, Soares LE, Martin AA, et al. Monomer conversion, microhardness, internal marginal adaptation, and shrinkage stress of bulk-fill resin composites. Dent Mater. 2015 Dec;31(12):1542-51. https://doi.org/10.1016/i.dental.2015.10.001

16. Van Ende A, Munck J, Lise DP, Van Meerbeek B. Bulk-fill composites: a review of the current literature. J Adhes Dent. 2017;19(2):95-109. https://doi.org/10.3290/i.jad.a38141

17. Ayres AP, Andre CB, Pacheco RR, Carvalho AO, Bacelar-Sá RC, Rueggeberg FA, et al. Indirect restoration thickness and time after light-activation effects on degree of conversion of resin cement. Braz Dent J. 2015 Jul-Aug;26(4):363-7. https://doi.org/10.1590/0103-64402013×0024

18. Al Sunbul H, Silikas N, Watts DC. Polymerization shrinkage kinetics and shrinkage-stress in dental resin-composites. Dent Mater. 2016 Aug;32(8):998-1006. https://doi.org/10.1016/i.dental.2016.05.006

19. Kalliecharan D, Germscheid W, Price RB, Stansbury J, Labrie D. Shrinkage stress kinetics of Bulk Fill resin-based composites at tooth temperature and long time. Dent Mater. 2016 Nov;32(11):1322-31. https://doi.org/10.1016/i.dental.2016.07.015

20. Veloso SR, Lemos CA, Moraes SL, Vasconcelos BCE, Pellizzer EP, Monteiro GQM. Clinical performance of bulk-fill and conventional resin composite restorations in posterior teeth: a systematic review and meta-analysis. Clin Oral Investig. 2019 Jan;23(1):221-33. https://doi.org/10.1007/s00784-018-2429-7

21. Chuang SF, Huang PS, Chen TY, Huang LH, Su KC, Chang CH. Shrinkage behaviors of dental composite restorations-The experimentalnumerical hybrid analysis. Dent Mater. 2016 Dec;32(12):e362-73. https://doi.org/10.1016/i.dental.2016.09.022

22. Rosatto CM, Bicalho AA, Veríssimo C, Bragança GF, Rodrigues MP, Tantbirojn D, et al. Mechanical properties, shrinkage stress, cuspal strain and fracture resistance of molars restored with bulk-fill composites and incremental filling technique. J Dent. 2015 Dec;43(12):1519-28. https://doi.org/10.1016/j.jdent.2015.09.007 
Pacheco RR, Garciaflores AF, Soto-Montero JR, Lesseux GG, Lancelotti ACRA, Martinez ED, et al.

23. Bicalho AA, Valdívia AD, Barreto BC, Tantbirojn D, Versluis A, Soares CJ. Incremental filling technique and composite material—-part II: shrinkage and shrinkage stresses. Oper Dent. 2014 Mar-Apr;39(2):E83-92. https://doi.org/10.2341/12-442-L

24. Kimura Y, Wilder-Smith P, Matsumoto K. Lasers in endodontics: a review. Int Endod J. 2000 May;33(3):173-85. https://doi.org/10.1046/j.1365-2591.2000.00280.x

25. Par M, Repusic I, Skenderovic H, Tarle Z. Wavelength-dependent light transmittance in resin composites: practical implications for curing units with different emission spectra. Clin Oral Investig. 2019 Dec;23(12):4399-409. https://doi.org/10.1007/s00784-019-02896-y

26. Tsai SR, Hamblin MR. Biological effects and medical applications of infrared radiation. J Photochem Photobiol B. 2017 May;170:197-207. https://doi.org/10.1016/i.jphotobiol.2017.04.014

27. Occhi-Alexandre IG, Baesso ML, Sato F, de Castro-Hoshino LV, Rosalen PL, Terada RS, et al. Evaluation of photosensitizer penetration into sound and decayed dentin: A photoacoustic spectroscopy study. Photodiagn Photodyn Ther. 2018 Mar;21(21):108-14. https://doi.org/10.1016/i.pdpdt.2017.11.008

28. Uo M, Kudo E, Okada A, Soga K, Kogo Y. Preparation and properties of dental composite resin cured under near infrared irradiation. J Photopolym Sci Technol. 2009;22(5):551-4. https://doi.org/10.2494/photopolymer.22.551

29. Mai HX, Zhang YW, Si R, Yan ZG, Sun LD, You LP, et al. High-quality sodium rare-earth fluoride nanocrystals: controlled synthesis and optical properties. J Am Chem Soc. 2006 May;128(19):6426-36. https://doi.org/10.1021/ja060212h

30. Iwamoto W, Vargas JM, Holanda Junior LM, Alves E, Moreno MS, Oseroff SB, et al. Improved route for the synthesis of colloidal NaYF4 nanocrystals and electron spin resonance of Gd3+ local probe. J Nanosci Nanotechnol. 2010 Sep;10(9):5708-14. https://doi.org/10.1166/jnn.2010.2438

31. Vert M, Doi Y, Hellwich KH, Hess M, Hodge P, Kubisa P, et al. Terminology for biorelated polymers and applications. Pure Ans Appl Chem. 2012;84(2):377-410. https://doi.org/10.1351/PAC-REC-10-12-04

32. Kong W, Sun T, Chen B, Chen X, Ai F, Zhu X, et al. A general strategy for ligand exchange on upconversion nanoparticles. Inorg Chem. 2017 Jan;56(2):872-7. https://doi.org/10.1021/acs.inorgchem.6b02479

33. Zhang H, Li Y, Lin Y, Huang Y, Duan X. Composition tuning the upconversion emission in NaYF4:Yb/Tm hexaplate nanocrystals. Nanoscale. 2011 Mar;3(3):963-6. https://doi.org/10.1039/c0nr00823k

34. Takagi H, Ogawa H, Yamazaki Y, Ishizaki A, Nakagiri T. Quantum size effects on photoluminescence in ultrafine Si particles. Appl Phys Lett. 1990;56(24):2379-80. https://doi.org/10.1063/1.102921

35. Shan J, Uddi M, Wei R, Yao N, Ju Y. The hidden effects of particle shape and criteria for evaluating the upconversion luminescence of the lanthanide doped nanophosphors. J Phys Chem C. 2010;114(6):2452-61. https://doi.org/10.1021/ip908976n

36. Krämer KW, Biner D, Frei G, Güdel HU, Hehlen MP, Lüthi SR. Hexagonal sodium yttrium fluoride based green and blue emitting upconversion phosphors. Chem Mater. 2004;16(7):1244-51. https://doi.org/10.1021/cm031124。

37. Harlow JE, Rueggeberg FA, Labrie D, Sullivan B, Price RB. Transmission of violet and blue light through conventional (layered) and bulk cured resin-based composites. J Dent. 2016 Oct;53:44-50. https://doi.org/10.1016/i.jdent.2016.06.007

38. Oberoi S, Zamperlini-Netto G, Beyene J, Treister NS, Sung L. Effect of prophylactic low level laser therapy on oral mucositis: a systematic review and meta-analysis. PLoS One. 2014 Sep;9(9):e107418. https://doi.org/10.1371/journal.pone.0107418

39. Scheps R. Upconversion laser processes. Prog Quantum Electron. 1996;20(4):271-358.

https://doi.org/10.1016/0079-6727(95)00007-0 